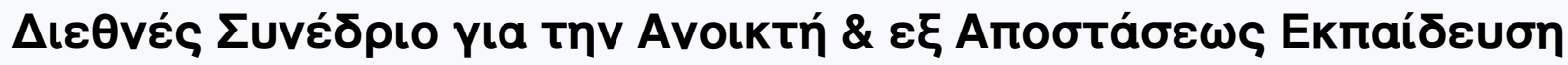

Tón. 6, Ap. 1A (2011)

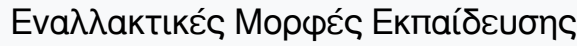

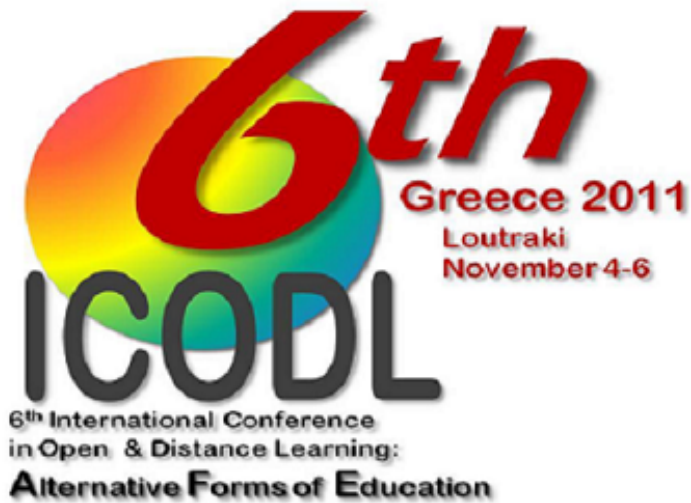

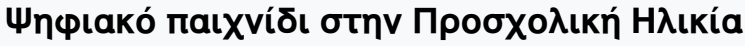

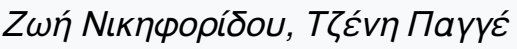

doi: $10.12681 /$ icodl. 679

TOMOEA

PART/ MEPOEA 


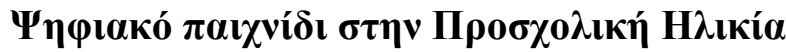

\section{Digital games in preschool education}

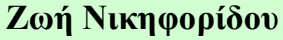

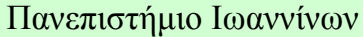 \\ $\Delta \mathrm{t} \delta \alpha ́ \kappa \tau \omega \rho \Pi \mathrm{TN}$ \\ znikifor@cc.uoi.gr
}

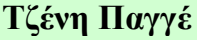

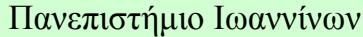 \\ К $\alpha \theta \eta \gamma \eta ́ \tau \rho ı ~ \Pi T N$ \\ jpagge@cc.uoi.gr
}

\begin{abstract}
The use of digital games as a contemporary educational practice is a new tool of learning, as long as it is exploited properly. Through an organized learning environment with educational goals, developmentally appropriate design and evaluation, digital games give learning a new dimension. The current paper stresses the main aspects of inclusion and integration of New Technologies and digital games in precise, in the preschool classroom. The general features of the digital games addressed to young children are depicted with emphasis on the pedagogical use, aiming at the development of knowledge, attitudes and ideas.
\end{abstract}

\section{Пєрі́ $\eta \psi \eta$}

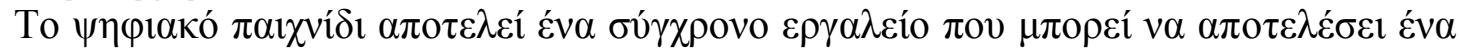

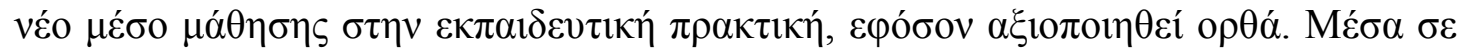

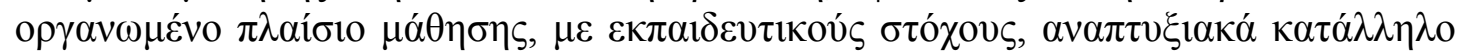

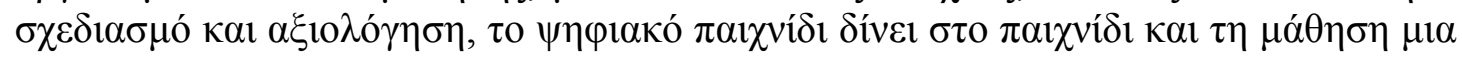

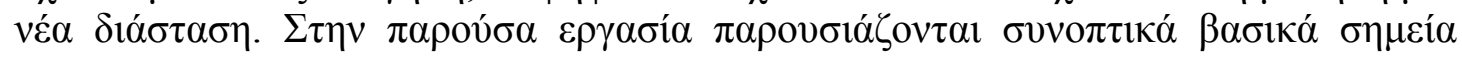

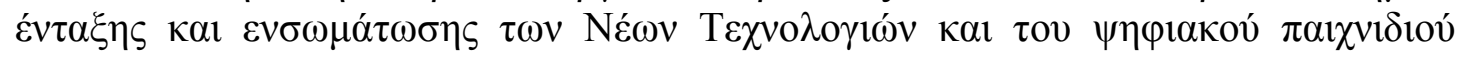

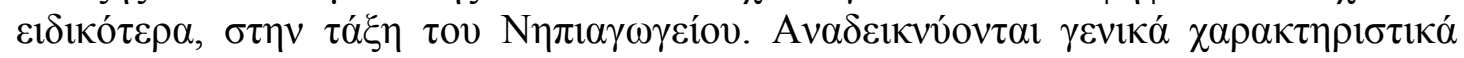

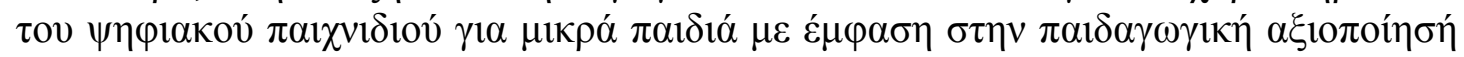

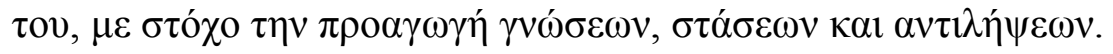

Key-words: $\psi \eta \varphi \imath \alpha \kappa o ́ ~ \pi \alpha \imath \chi v i \delta l, \pi \rho o \sigma \chi o \lambda \imath \kappa \eta ́ ~ \eta \lambda \imath \kappa i \alpha$

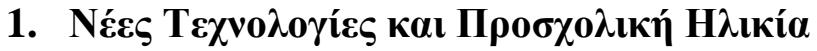

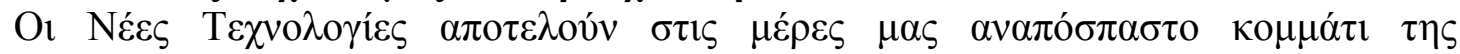

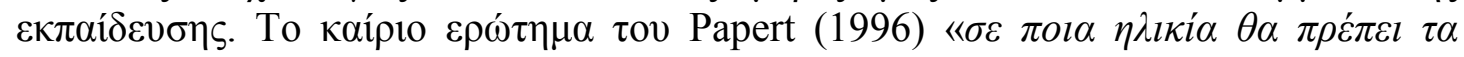

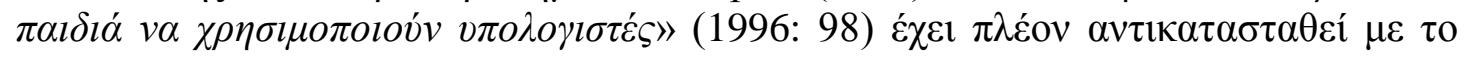

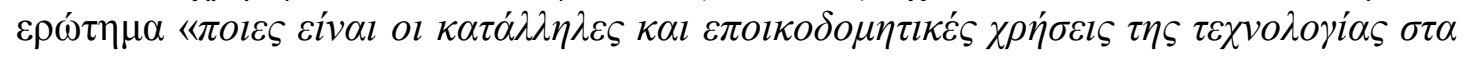

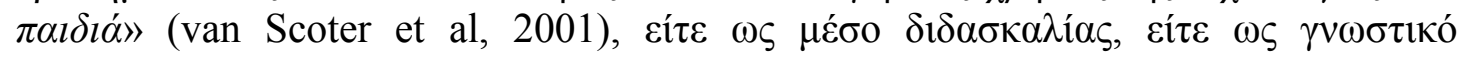

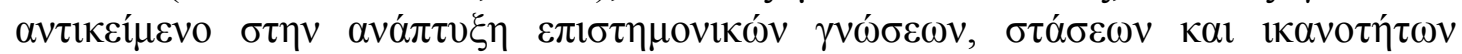

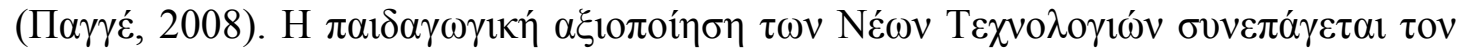

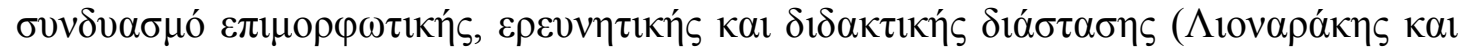
Фраүка́кๆ, 2009).

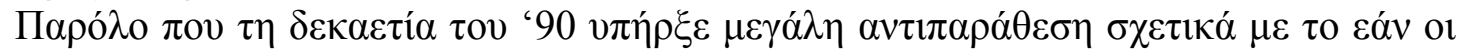

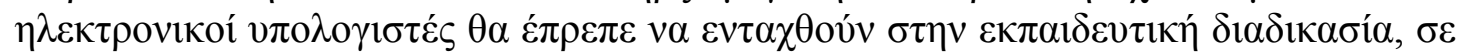

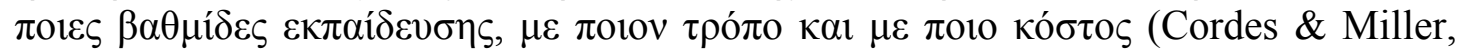

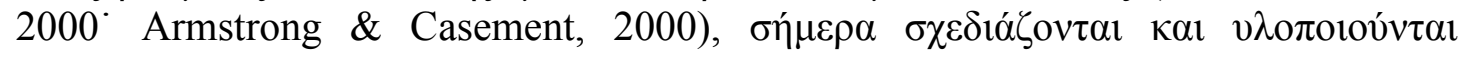




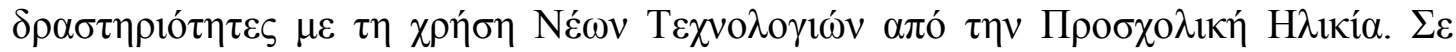

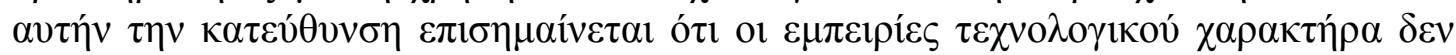

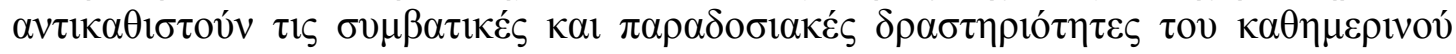

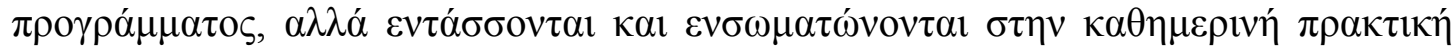

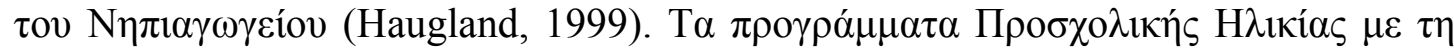

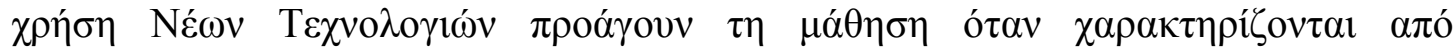

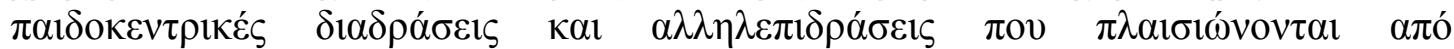

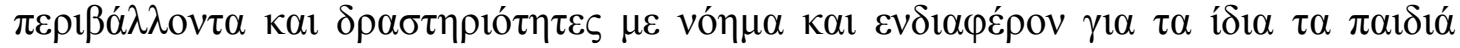
(Yelland, 2005; Siraj-Blatchford \& Siraj-Blatchford, 2006; Stephen \& Plowman, 2003).

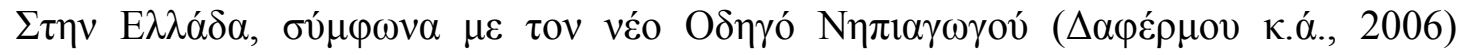

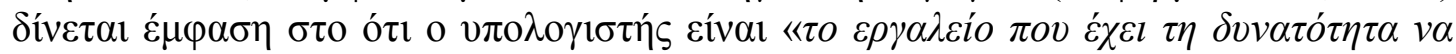

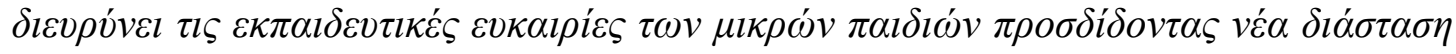

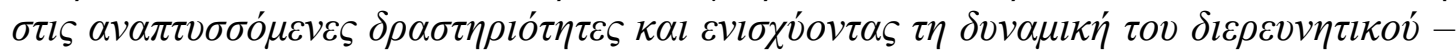

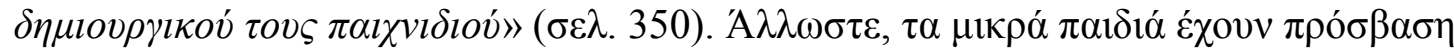

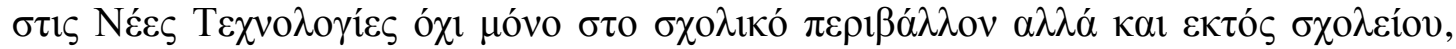

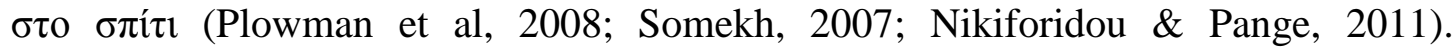

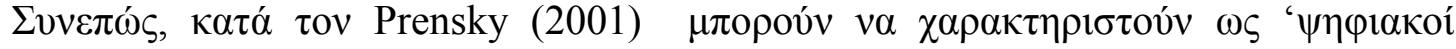

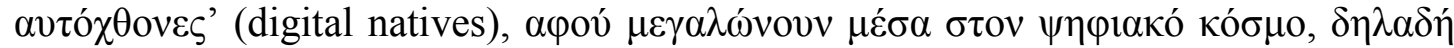

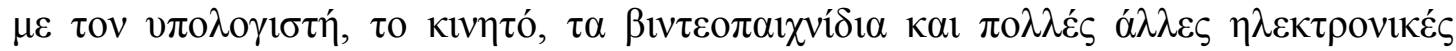

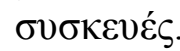

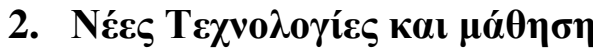

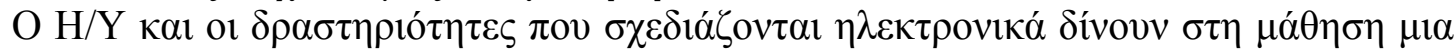

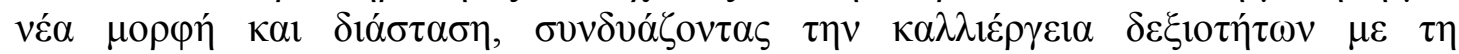

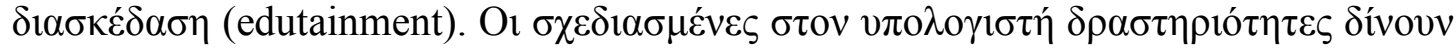

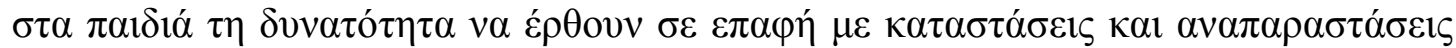

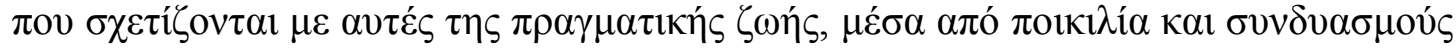

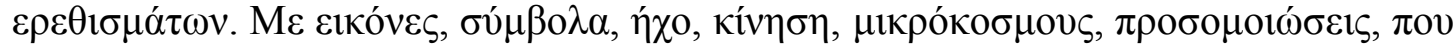

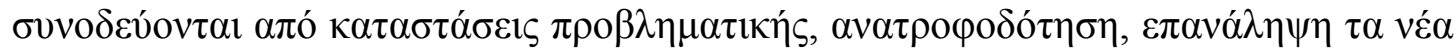

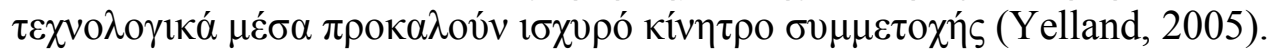

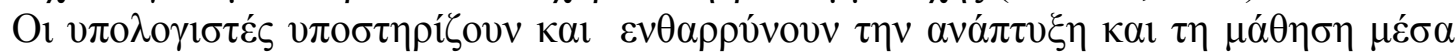

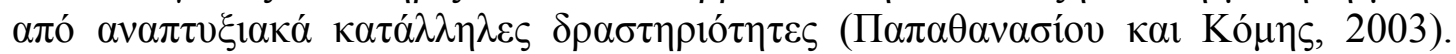

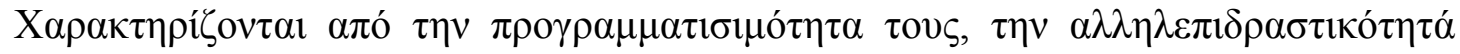

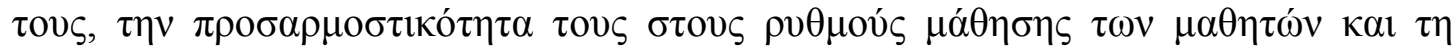

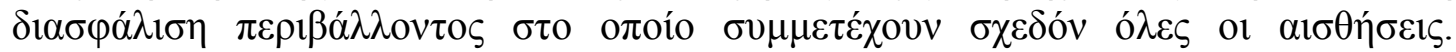

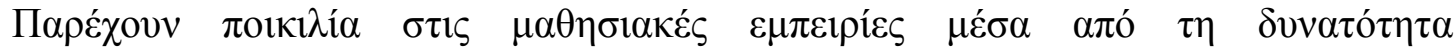

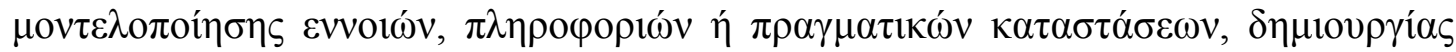

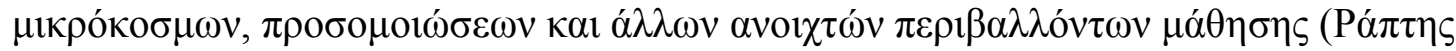
\& Ра́ $\pi \tau \eta, 2006)$.

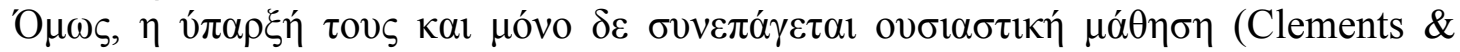

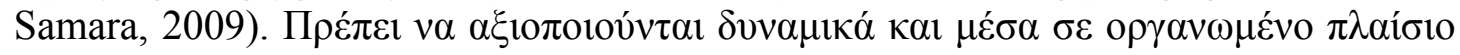

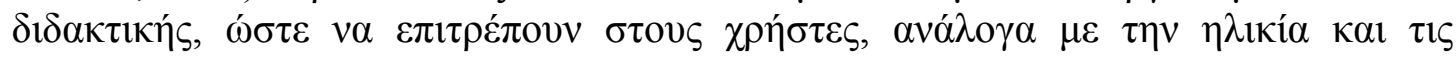

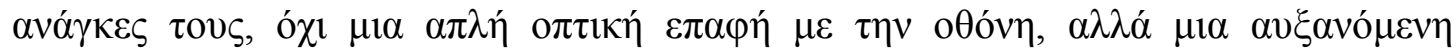

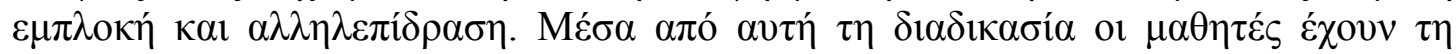

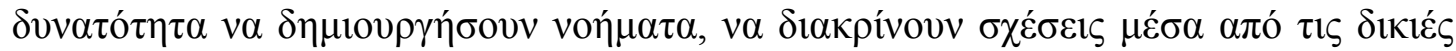

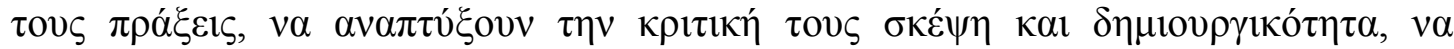

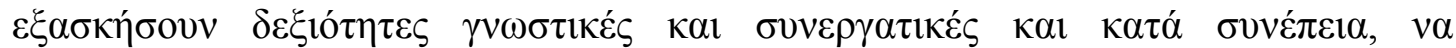

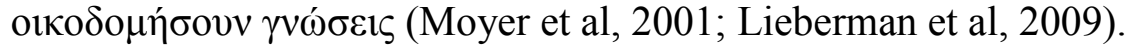




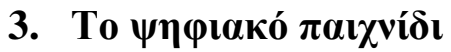

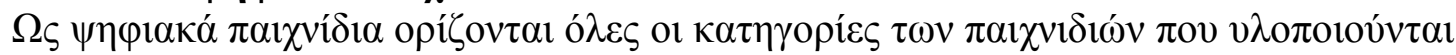

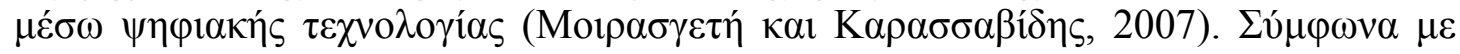

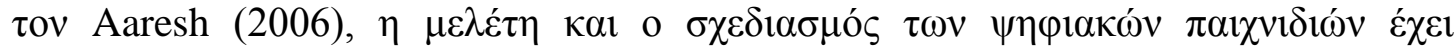

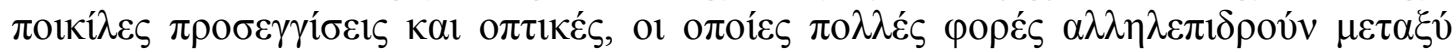

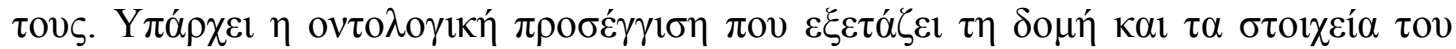

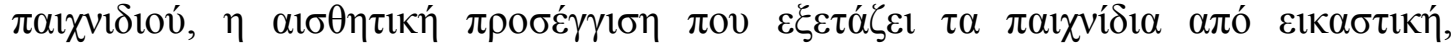

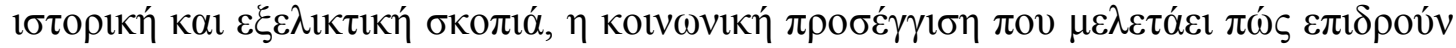

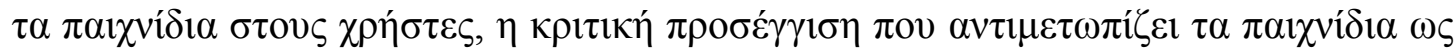

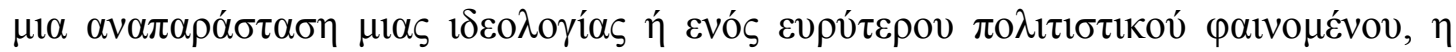

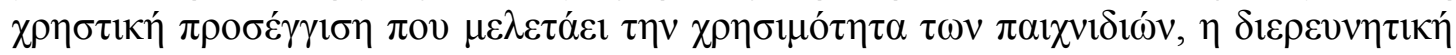

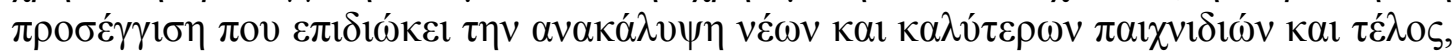

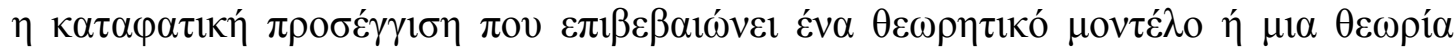

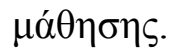

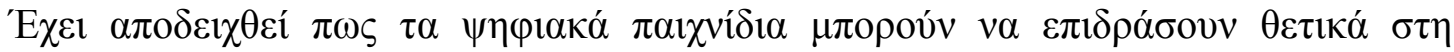

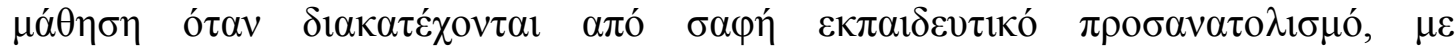

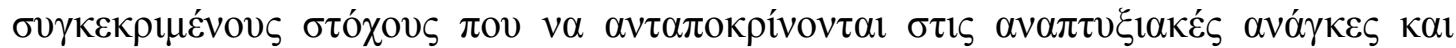

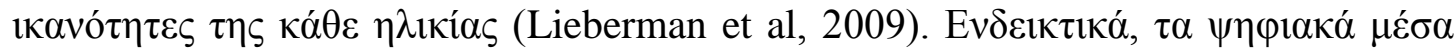

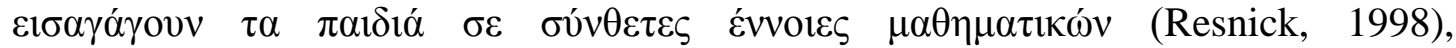

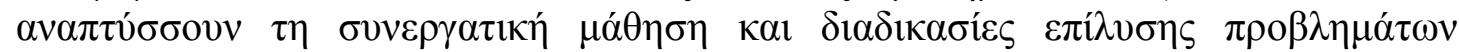

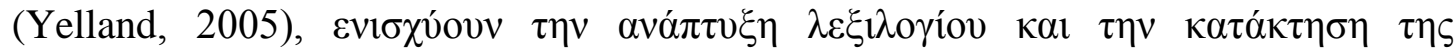

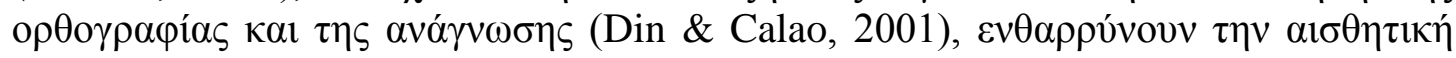

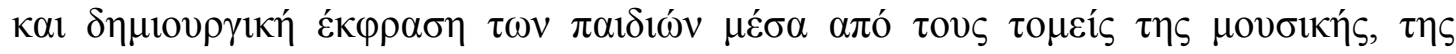

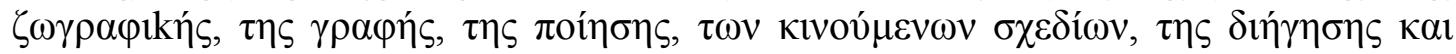

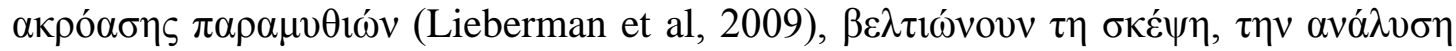

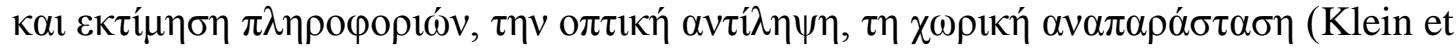

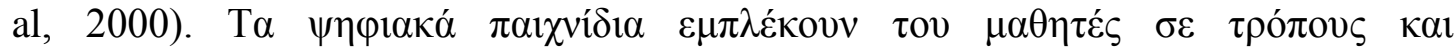

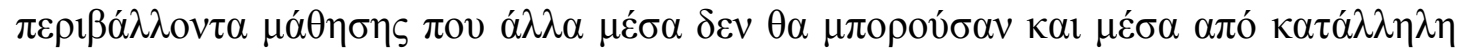

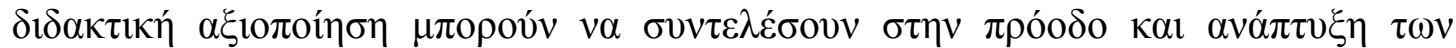
$\pi \alpha 1 \delta i \omega ́ v$.

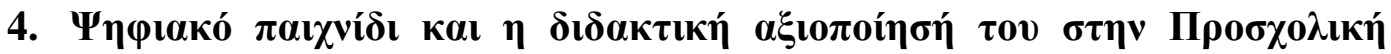 Н $\lambda$ uкía}

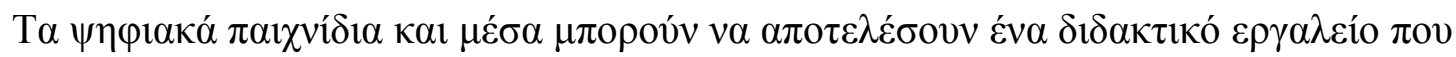

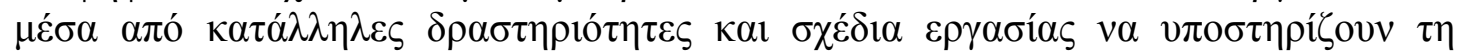

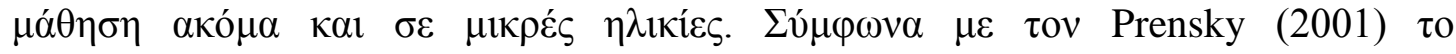

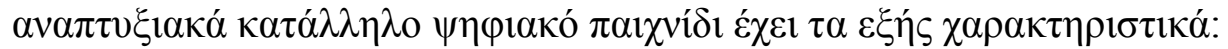

1. Kavóves

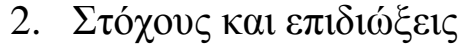

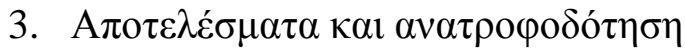

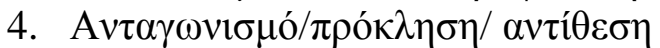

5. $А \lambda \lambda \eta \lambda \varepsilon \pi i \delta \rho \alpha \sigma \eta$

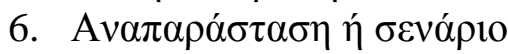

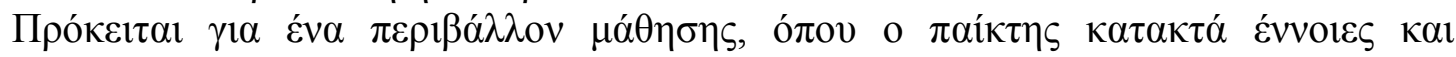

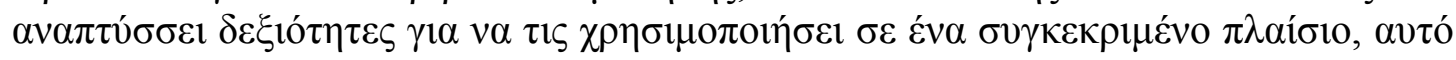

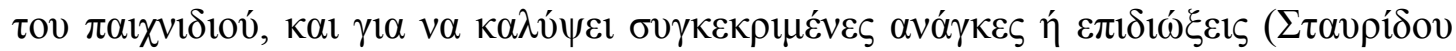

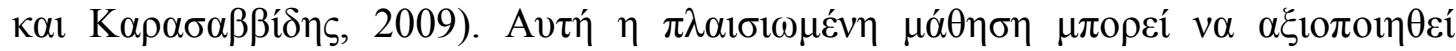

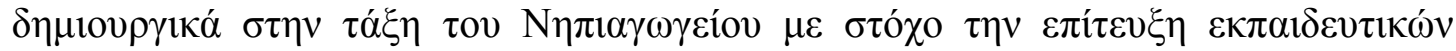

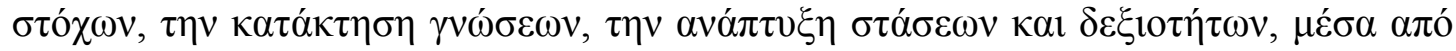

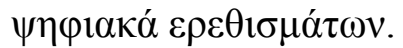




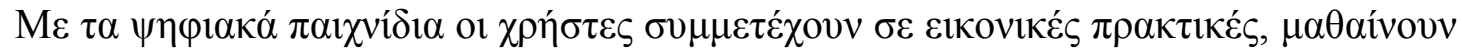

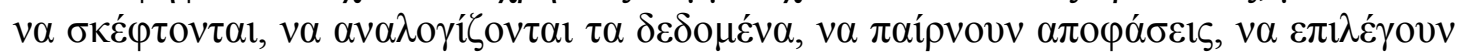

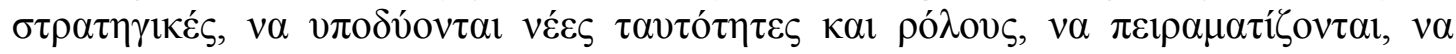

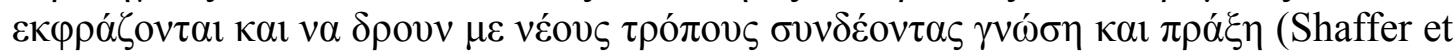

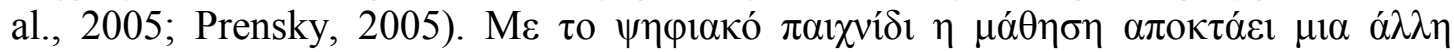

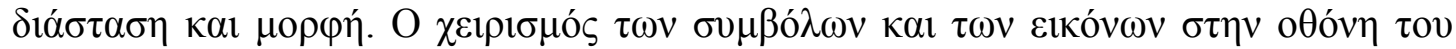

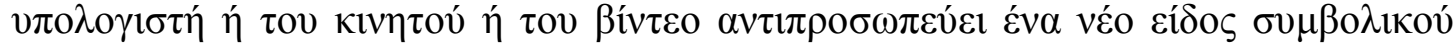

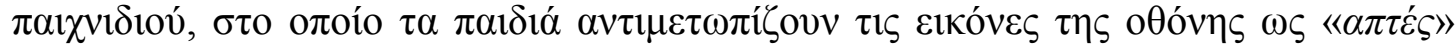

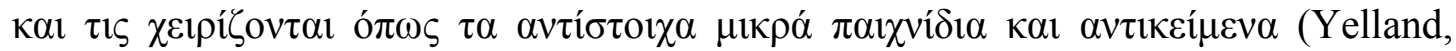

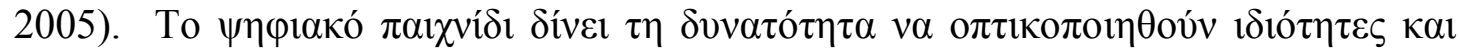

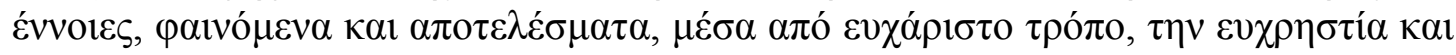

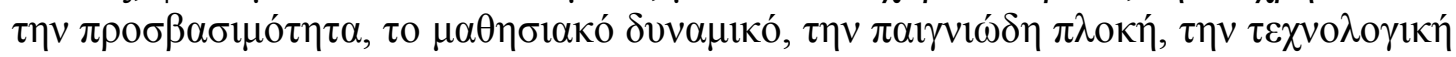

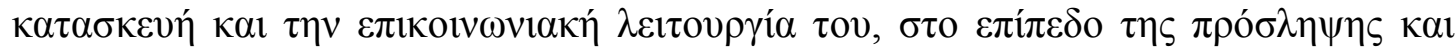

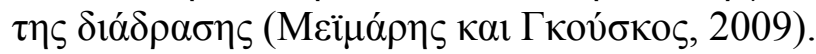

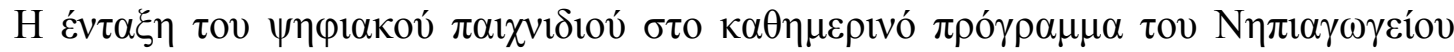

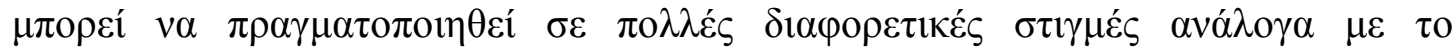

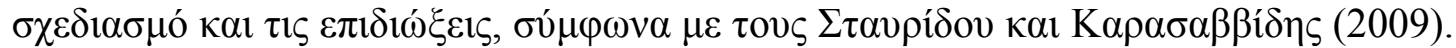

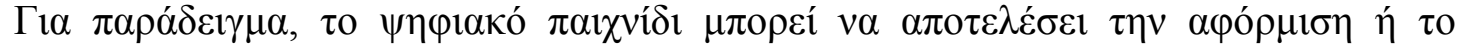

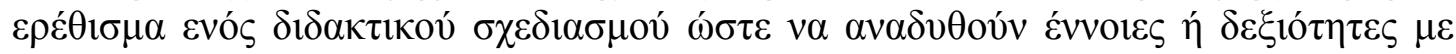

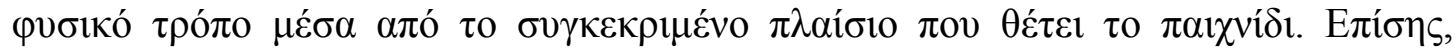

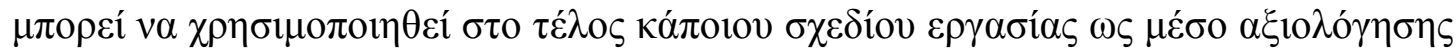

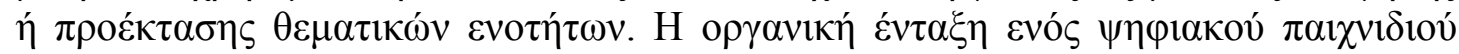

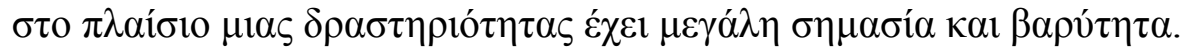

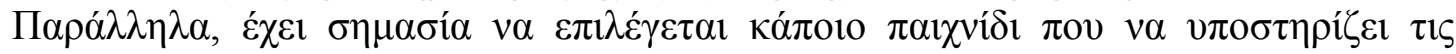

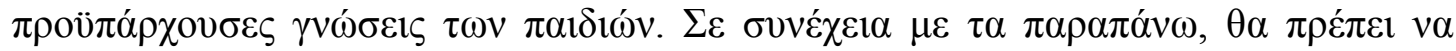

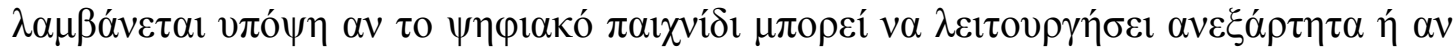

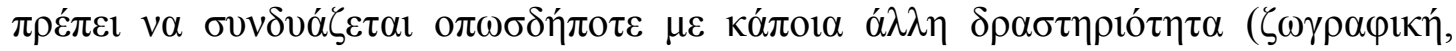

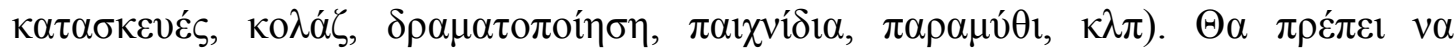

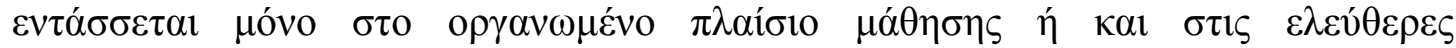

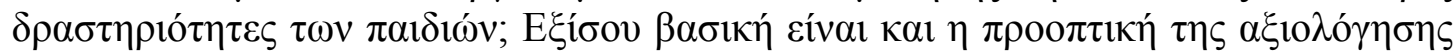

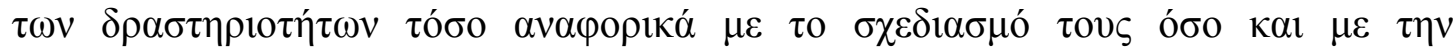

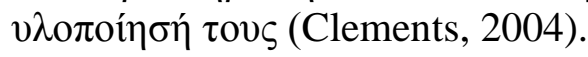

\section{5. $\Sigma v \mu \pi \varepsilon \rho \alpha ́ \sigma \mu \alpha \tau \alpha$}

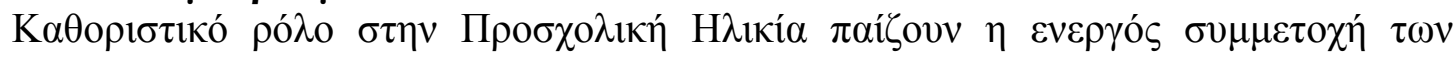

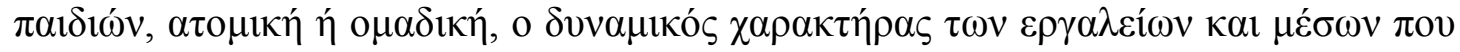

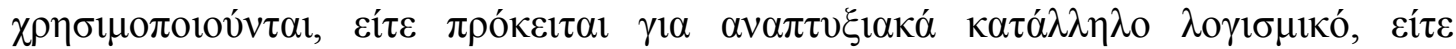

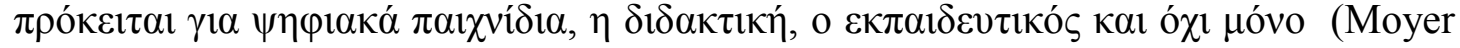

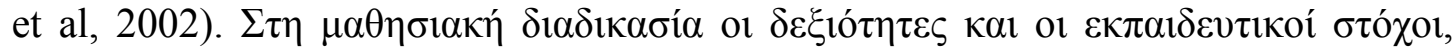

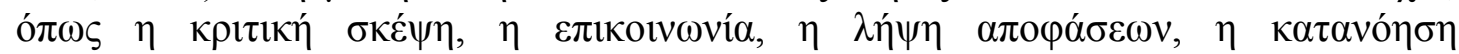

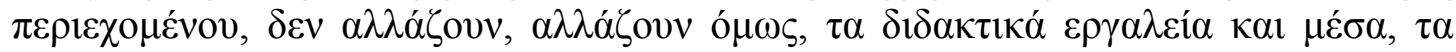

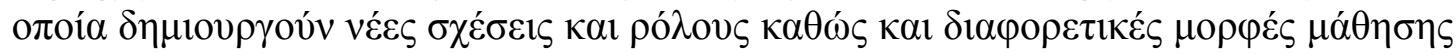

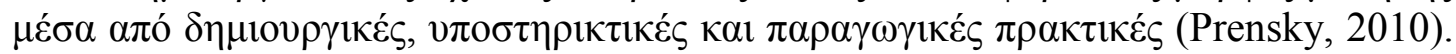

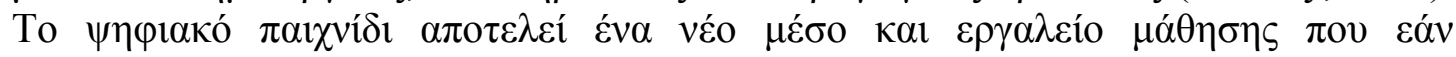

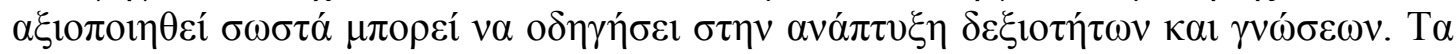

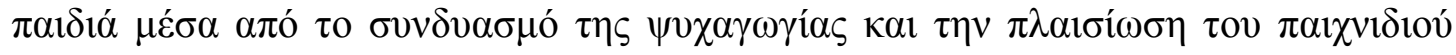

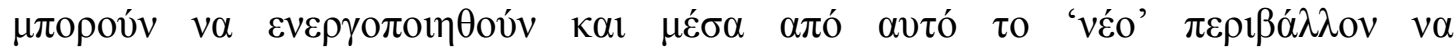

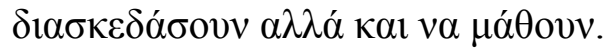




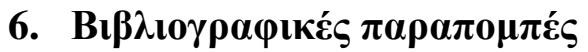

Aaresh, E. (2006). Mapping the Madness: A Games Analysis Framework. From Games to Gaming RResearch Seminar. Gothenburg University.

Armstrong, A. \& Casement, C. (2000). The child and the machine. Why computers may put our children's education at risk. Beltville: Robins Lane Press.

Clements, D. \& Sarama, J. (2009), Learning and teaching early math: The learning trajectories approach, New York: Routledge.

Clements, D. (2004). Major themes and recommendations. In D. H. Clements, J. Sarama, \& A. DiBiase (Eds.), Engaging young children in mathematics: Standards for early childhood mathematics education (pp. 7-72). New Jersey: Lawrence Erlbaum.

Cordes, C. \& Miller, E. (Eds.) (2000). Fool's gold: A critical look at computers in childhood. College Park, MD: Alliance for Childhood.

Din, F.S. \& Calao, J. (2001). The effects of playing educational video games on kindergarten achievement. Child Study Journal, 31(2), pp. 95-102.

Haugland, S. (1999). What role should technology play in young children's learning? Young Children, 54(6), pp. 26-31.

Klein, P., Nir-Gal, O., \& Darom, E. (2000). The use of computers in kindergarten with or without adult mediation; effects on children's cognitive performance and behaviour. Computers in Human Behaviour, 16, pp. 591-608.

Lieberman D., Bates, C., Jiyeon S. (2009). Young children's learning with digital media. Computers in the schools, 26, pp.271-283

Moyer, P. S., Bolyard, J. J., \& Spikell, M. A. (2002). What are virtual manipulatives? Teaching Children Mathematics, 8(6), pp. 372-377.

Nikiforidou, Z. and Pange, J. (2010). Teachers' evaluation of preschool educational software: the case of probabilistic thinking. Procedia - Social and Behavioral Sciences 9, pp. 537-541

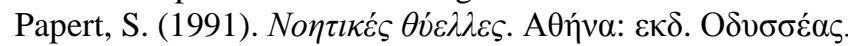

Plowman, L., \& Stephen, C. (2005). Children, play, and computers in pre-school education. British Journal of Educational Technology, 36 (2), pp. 145-157.

Prenksy, M. (2001). Digital natives, digital immigrants. On the Horizon, 9, 5, 1-6.

Prensky, M. (2005). "Engage Me or Enrage Me": What Today's Learners Demand. EDUCAUSE Review, 40(5), pp. 60-65.

Prensky, M. (2003). Digital game-based learning. ACM Computers in Entertainment, 1(1), 21.

Prensky, M. (2010). Teaching Digital Natives, Partnering for Real Learning Thousand Oaks: Corwin.

Resnick, M., Berg, R., and Eisenberg, M. (2000). Beyond Black Boxes: Bringing Transparency and Aesthetics Back to Scientific Investigation. Journal of the Learning Sciences, vol. 9, no. 1, pp. 7-30.

Shaffer, D.W., Squire, K.R., Havelson, R. \& Gee, J.P. (2005). Video games and the future of learning. Phi Delta Kappan, 87 (2), pp. 104-111.

Siraj-Blatchford, I., \& Siraj-Blatchford, J. (2006). A curriculum development guide to ICT in Early Childhood Education, Nottingham: Trentham Books with Early Education.

Somekh, B. (2007) Pedagogy and Learning with ICT: researching the art of innovation. London and New York: Routledge.

Plowman, L. \& C. Stephen (2003). A 'benign addition'? Research on ICT and pre-school children. Journal of Computer-Assisted Learning. 19 (2), pp. 149-164.

Van Scoter, J., Ellis, D. \& Railsback, J. (2001). How Technology Can Enhance Early Childhood Learning, $\quad$ Retrieved on $20 \quad$ February, 2011 from http://www.netc.org/earlyconnections/byrequest.html

Yelland, N. (2005). The future is now: A review of the literature on the use of computers in early childhood education (1994-2004). AACE Journal, 13(3), pp. 201- 232.

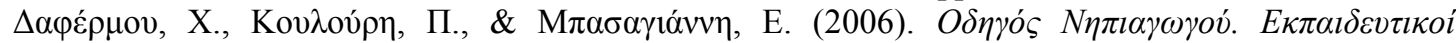

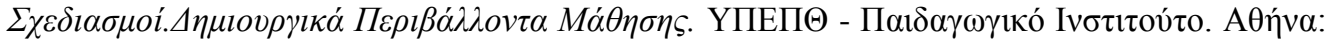
$\mathrm{OE} \Delta \mathrm{B}$.

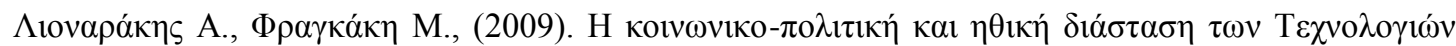

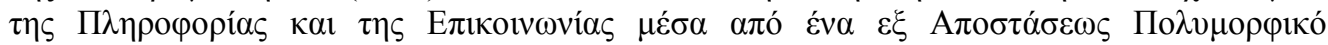

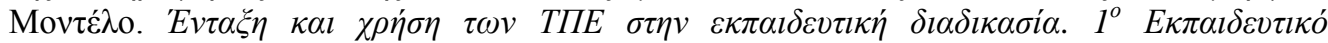
$\Sigma v v \varepsilon ́ \delta \rho \imath o, ~ \Pi \alpha v \varepsilon \pi 1 \sigma \tau \eta \dot{\mu l o} \Theta \varepsilon \sigma \sigma \alpha \lambda i ́ \alpha$.

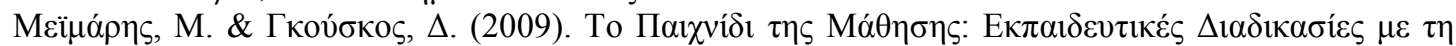

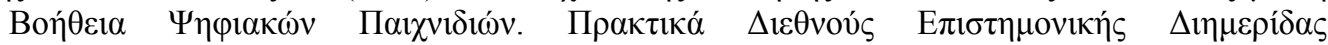

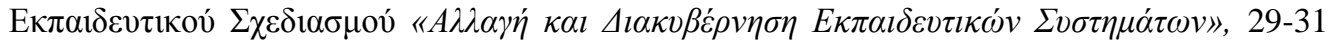

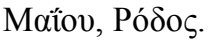




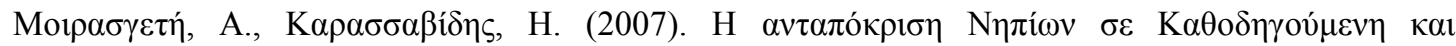

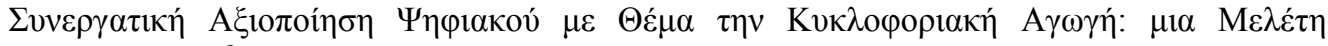

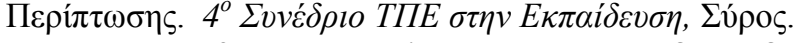

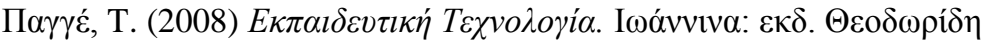

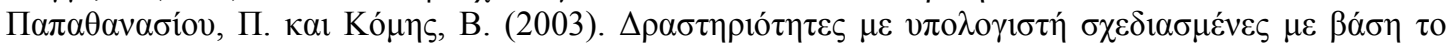

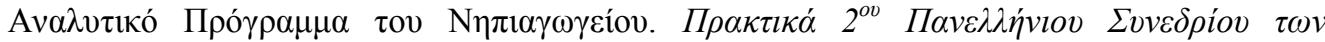

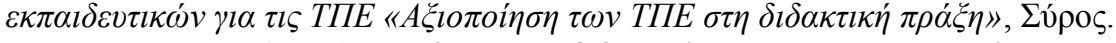

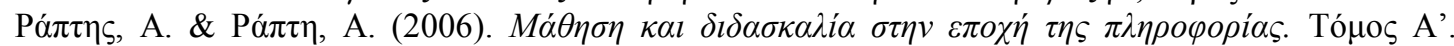
A $\theta \eta \dot{v \alpha: ~ \alpha v \tau о \varepsilon ́ \kappa \delta o \sigma \eta . ~}$

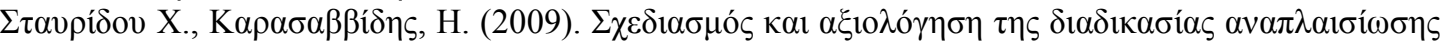

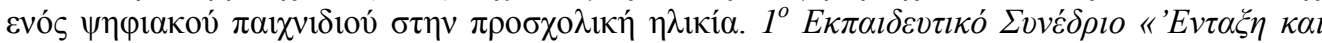

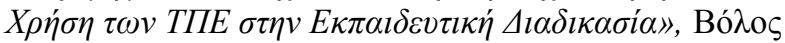

